\title{
A simple approach to the evaluation of fiber/matrix interfacial shear strength and fracture toughness
}

\author{
S.Y. Zhang* \\ Institute of Mechanics, Chinese Academy of Sciences, Beijing 100080, China
}

Received 10 May 1999; accepted 30 July 1999

\begin{abstract}
On the basis of microscopical analyses of the fiber distribution and longitudinal shear deformation in unidirectional fiber composites, a simple approach is presented for characterizing the interfacial shear strength and fracture toughness. C 2000 Elsevier Science Ltd. All rights reserved.
\end{abstract}

Keywords: Microscopical analysis; Interfacial shear strength; Interfacial fracture toughness; Unidirectional fiber composite

\section{Introduction}

The fiber/matrix interfacial mechanical properties, especially the interfacial shear strength and fracture toughness, influence the macroscopic properties of composite materials and they have attracted much attention. In 1993, a round-robin program [1] was undertaken to investigate testing methods of measuring the interfacial shear strength. The four commonly used methods, i.e. single-fiber pull-out, microdebonding, fragmentation, and micro-indentation tests, were selected. The results show a high level of scatter, and the existing methods are not therefore very reliable: a new method needs to be developed.

There are several test methods for evaluating the interfacial fracture toughness of the bi-material interface [2]. To evaluate the interfacial fracture properties of the fiber/matrix interface, the most pertinent method is the single-fiber pull-out test of the concentrated axisymmetric model [3]. This method is simple in principle. However, since the fiber is so thin, many factors affect the accuracy of the results and the embedded length and the free length of the fiber are difficult to measure exactly, so the results show a large scatter [4]. On the basis of microscopical analysis of the fiber distribution and shear deformation, the present author proposes a new method for characterizing the interfacial bond

* Fax: + 86-10-6256-1284

E-mail address: syzhang@imech.ac.cn (S.Y. Zhang). strength and fracture toughness, which is easy to perform and results in reasonable predictions.

\section{Microscopical geometric analysis of fiber distributions in unidirectional composites}

Assuming the fibers are distributed hexagonally, through a simple geometrical analysis the following relationship is obtained:

$s=2\left[\left(\frac{\pi}{2 \sqrt{3} v_{f}}\right)^{\frac{1}{2}}-1\right] r$

where $v_{f}$ is the volume fraction of the fiber, $s$ is the distance between two fibers, and $r$ is fiber radius. For square distribution of the fibers, Eq. (1) changes to:

$s=2\left[\left(\frac{\pi}{4 v_{f}}\right)^{\frac{1}{2}}-1\right] r$

The distance between the centers of two fibers is: $S=2 r+s$.

Because the fiber modulus is much larger than that of the matrix, i.e. the modulus of glass fiber is larger than that of epoxy by an order of magnitude, when the composite undergoes a longitudinal shear deformation, the fiber modulus can be assumed to be infinitely large 
and the shear deformation is all caused by the matrix. Thus, the average shear strain is given as:

$\gamma_{\mathrm{av}}=\frac{\Delta u}{s+2 r}$

where $\Delta u$ is the difference between the longitudinal displacement of the centers of the two adjacent fibers in the through-thickness direction, i.e. $\Delta u=u_{02}-u_{01}$ (Fig. 1). In the matrix, the maximum shear strain takes place at the location where the separation distance of the two fibers is the shortest, as shown by $\overline{A_{1} A_{2}}$ in Fig. 1. Thus the maximum shear strain in the matrix is given as:

$\gamma_{\max }=\frac{\Delta u}{s}$

Consequently, the relationship between the average shear strain of the composite and the maximum strain in matrix is derived as:

$\frac{\gamma_{\mathrm{av}}}{\gamma_{\max }}=\frac{s}{s+2 r}$

\section{Measurement of the interfacial shear strength}

The method of measuring the interlaminar shear strength by the short-beam bending test has been well documented. The interlaminar shear stress (ILSS) in the short-beam bending test is given as:

$\tau_{\mathrm{il}}=\frac{3 p}{4 b h}$

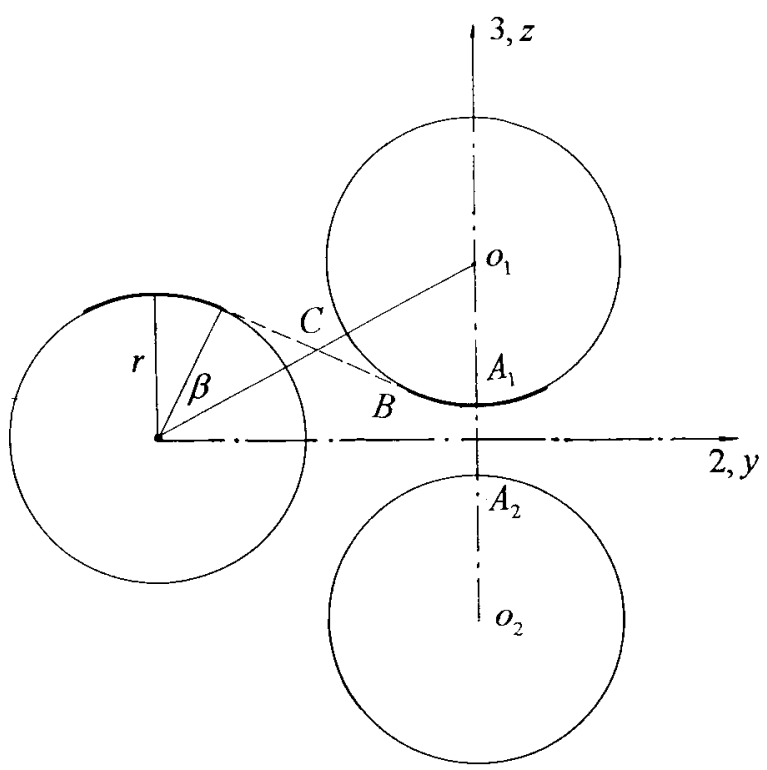

Fig. 1. Hexagonal fiber distribution and interfacial crack. where $p$ is bending load, $b$ is width, and $h$ is thickness of the beam. The ILSS $\left(\tau_{\mathrm{il}}\right)$ in Eq. (6) is that in the middle surface of the beam, and it is the apparent average stress. According to Eq. (3), we have:

$\tau_{\mathrm{il}}=G_{13 c} \gamma_{\mathrm{av}}=G_{13 c} \frac{\Delta u}{s+2 r}$

where $G_{13 c}$ is the principal (longitudinal-transverse) shear modulus of the composite, and subscripts 1 and 3 refer to fiber direction and transverse direction, respectively (Fig. 1). The maximum shear stress in the matrix is:

$\tau_{\max }=G_{\mathrm{m}} \gamma_{\max }=G_{\mathrm{m}} \frac{\Delta u}{s}$

Consequently,

$\tau_{\max }=\tau_{\mathrm{il}} \frac{G_{\mathrm{m}}}{G_{13 c}} \times \frac{s+2 r}{s}$

Thus, after measurement of the interlaminar shear strength, $\tau_{\mathrm{il}}^{c}$, from Eq. (9) the shear strength of the interface (or matrix) can be obtained. If the shear strength of the matrix is larger than that of the interface, Eq. (9) gives the interfacial shear strength, otherwise it is matrix strength.

The shear modulus, $G_{13 c}$, of unidirectional fiber composites can be determined by the role of mixtures. However, the following formula yields better predictions [5]:

$G_{13 c}=\frac{G_{m}}{1-\sqrt{v_{f}}\left(1-G_{m} / G_{13 f}\right)}$

Taking glass-fiber-reinforced low-modulus epoxy as an example, the matrix modulus is, $G_{m}=0.77 \mathrm{GPa}$, the modulus of glass fiber, $G_{13 f}=30 \mathrm{GPa}$, and we assume that $v_{f}=0.6$. Thus Eq. (9) gives $\tau_{\max }=1.31 \tau_{\mathrm{il}}$. Compared with the experimental results of [6], this proved to be a good prediction.

\section{Measurement of interfacial fracture toughness}

The end-notched-beam flexure (ENF) test is a commonly used method for the evaluation of interlaminar shear (mode II) fracture toughness, $G_{\mathrm{II}}^{c}$. The formula is given as [7]:

$G_{\mathrm{II}}^{c}=\frac{9 a^{2} p_{c}^{2}}{b^{2} h^{3} E_{11}}$

where $E_{11}$ is longitudinal modulus, $p_{c}$ is fracture load of the ENF test, $a$ is crack length $b$ is width, and $h$ is a half of the thickness of the beam. Considering the shear effect the expression is modified to be [7]: 
$G_{\mathrm{II}}^{c}=\frac{9 a^{2} p_{c}^{2}}{b^{2} h^{3} E_{11}}\left[1+0.2 \frac{E_{11}}{G_{13}}\left(\frac{h}{a}\right)^{2}\right]$

The critical stress intensity factor is given as:

$K_{\mathrm{II}}^{c}=\tau_{\mathrm{c}}(\pi a)^{1 / 2} H$

where $\tau_{c}$ is the apparent fracture shear stress determined by (6) and

$$
\begin{aligned}
H\left(\frac{a}{2}\right)^{\frac{1}{2}}= & 0.368-0.0717\left(\frac{e}{d}\right)+0.0045\left(\frac{e}{d}\right)^{2}+0545\left(\frac{a}{e}\right) \\
& +0.490\left(\frac{e}{d}\right)\left(\frac{a}{e}\right)-0.0209\left(\frac{a}{e}\right)\left(\frac{e}{d}\right)^{2}
\end{aligned}
$$

where the parameters in the above expression are shown in Fig. 2. From fracture mechanics, we have:

$G_{\mathrm{II}}^{c}=\frac{S_{11}}{\sqrt{2}}\left[\left(\frac{S_{33}}{S_{11}}\right)^{\frac{1}{2}}+\frac{2 S_{13}+S_{55}}{2 S_{11}}\right]^{\frac{1}{2}} \times K_{\mathrm{II}}^{c^{2}}$

where $S_{i j}$ is compliance tensor of the composite, and 1, 3 indicate longitudinal and through thickness directions, respectively.

For unidirectional fiber composites, the fracture surface of the interlaminar crack is microscopically uneven. The interfacial crack surface of the fiber/matrix is a partial cylindrical (or circle arc) surface and they are linked by matrix cracks. The lengths of the matrix crack and of the interfacial crack are determined by the difference of the matrix strength and interfacial strength. Higher strength leads to shorter crack length. The simplest situation is that the matrix crack is a tangent to interfacial cracks of the adjacent fibers (see Fig. 1 for the hexagonal distribution). From the geometry of Fig. 1, the lengths of the two cracks can be calculated.

The length of a half interfacial crack is:

$l_{i}=r\left(\frac{\pi}{3}-\beta\right)$

where the angle $\beta$ is determined by:

$\cos ^{-1} \beta=\frac{r}{r+s / 2}$

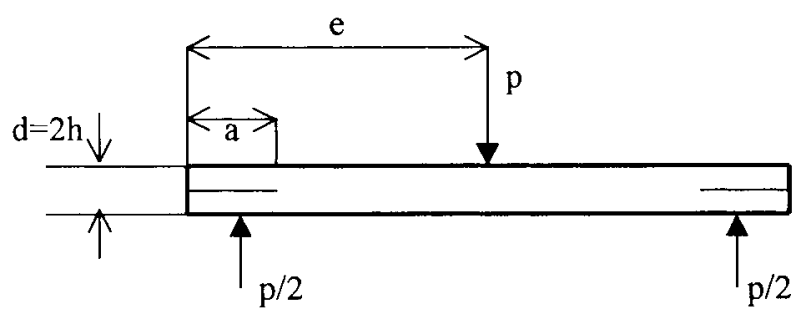

Fig. 2. Schematic showing ENF specimen.
The length of a half matrix crack $l_{m}$ is:

$l_{m}=r \tan (\beta)$

For $v_{f}=0.6$, it is obtained that $l_{i}=0.43 r$ and $l_{m}=0.71 r$.

The interlaminar fracture toughness of composite, $G_{I I}^{c}$, consists of two parts: contributions of matrix crack and interfacial crack. According to the rule of mixtures, we have:

$G_{\mathrm{II}}^{c}=\frac{1}{l_{i}+l_{m}}\left(l_{i} G_{\mathrm{II}}^{i}+l_{m} G_{\mathrm{II}}^{m}\right)$

where $G_{\mathrm{II}}^{i}$ is the critical strain-energy release rate (fracture toughness) for interfacial fracture. $G_{I I}^{m}$ is critical strain-energy release rate of the matrix. After $G_{I I}^{c}$ is measured by the ENF test and $G_{I I}^{m}$ is known from handbooks of materials properties. $G_{\mathrm{II}}^{i}$ can be calculated from (17). As an example, a T300 carbon fiber reinforced XU292 resin composite is considered. From the handbook, it is known that the fracture toughness of the resin XU292 is: $G_{\mathrm{II}}^{m}=210.0 \mathrm{~J} / \mathrm{m}^{2}(1.2 \mathrm{in}-\mathrm{lb} / \mathrm{in})$. The measured data give $G_{\mathrm{II}}^{c}=1.48 \mathrm{~kJ} / \mathrm{m}^{2}$; thus, Eq. (17) gives $G_{\mathrm{II}}^{i}=3.57 \mathrm{~kJ} / \mathrm{m}^{2}$. It is easy to see that the interfacial debonding crack absorbs more energy than the matrix crack. This is why fiber composites possesses much larger fracture toughness than matrix.

The above discussion is given for the case of a hexagonal distribution of the fibers. For the square fibre distribution, longer interfacial cracks may be expected if the interfacial strength is smaller than that of the matrix. In the limit, it is assumed that $l_{i}=\frac{\pi}{2} r$ and $l_{m}=\frac{s}{2}=0.288 r$, and we obtain $G_{\mathrm{II}}^{i}=1.71 \mathrm{~kJ} / \mathrm{m}^{2}$, which is approximately equal to $G_{I I}^{c}$.

Furthermore, this method can also be used to evaluate the Mode I fracture toughness for interfacial cracking by using delamination fracture tests. For example, one can use the double-cantilever bend (DCB) test to measure Mode I fracture toughness $G_{\mathrm{I} c}$ of composites. From Eq. (17), the interfacial fracture toughness can be estimated. If one carries out mixed-mode bending (MMB) tests [8], then the mixed-mode fracture toughness can also be obtained.

\section{Conclusions}

A simple method is presented for evaluating the interfacial strength and fracture toughness of fiber composites. In principle, this method cannot produce exact experimental values. However, it is simple and able to obtain reasonable estimation, especially, for the case that differences between the interfacial strength and fracture toughness and those of the matrix are not too large. 


\section{Acknowledgement}

This paper has been supported by the Chinese Science Foundation.

\section{References}

[1] Pitkethly MJ, et al. A round-robin program on interfacial test methods. Comp Sci Technol 1993;48:205-14.

[2] Cao HC, Evans AG. An experimental study of the fracture resistance of bimaterial interfaces. Mechanics of Materials 1989;7:295-304.

[3] Zhang SY. A new model for the energy release rate of fiber/ matrix interfacial fracture. Comp Sci Technol 1998;58:163-6.
[4] Piggott MR. Debonding and friction at fiber-polymer interfaces. I: criteria for failure and sliding. Comp Sci Technol 1987;30:295306.

[5] Chamis CC. Simplified composite micromechanics equations for strength, fracture toughness and environmental effects. 39th Annual Conf, Reinf Plastics and Comp Inst, 16-19 January 1984.

[6] Hull D. An introduction to composite materials. Cambridge University Press, 1981. p. 48-57.

[7] Grady JE. Fracture toughness testing of polymer matrix composites. In: Cheremisinoff NP, editor. Handbook of ceramics and composites, vol. 2. Marcel Dekker Inc., 1992. p. 1-50.

[8] Reeder JR, Crews Jr JH. Mixed-mode bending method for delamination testing. AIAA J 1990;28(7):1270-6. 